\title{
247. On an Algebraic Model for von Neumann Algebras. II
}

\author{
By Hiroshi TAKaI \\ Department of Mathematics, Osaka Kyoiku University \\ (Comm. by Kinjirô KunUgr, M. J. A., Dec. 12, 1970)
}

1. In the previous paper [6], we have introduced algebraic models of von Neumann algebras as a non-commutative extension of algebraic models of measures due to Dinculeanu and Foias [2], and we have proved that an algebraic model determines the algebra structure up to isomorphisms (unfortunately, a von Neumann algebra can not decide an algebraic model within isomorphisms).

In an another note [3], Dinculeanu and Foiaş, introduced an another concept, that is, algebraic models for measure preserving transformations. In $\S 2$, we shall extend the above notion to an automorphism of von Neumann algebra. In $\S 3$, we shall present a measure system which is an algebraic model for the crossed product of a von Neumann algebra by an automorphism group. And in $\S 4$, we shall present an algebraic ergodic system which is an algebraic model for a certain automorphism of the crossed product.

Dinculeanu and Foias [3] introduced also the notion of discrete models of measures and established several important theorems. A non-commutative variant of discrete models gives a characterization of group von Neumann algebras. We shall discuss discrete models in a subsequent paper.

Throughout the note, we shall use the terminology of [4] without explanations.

2. Let $(\Gamma, \varphi)$ be a measure system introduced in [6], $U$ be an isomorphism of $\Gamma$ into $\Gamma$. Then we shall say $(\Gamma, U, \varphi)$ an algebraic ergodic system if

$$
\varphi(U \gamma)=\varphi(\gamma) \quad \text { for every } \quad \gamma \in \Gamma
$$

Two algebraic ergodic systems $(\Gamma, U, \varphi)$ and $(\Delta, V, \varphi)$ are called isomorphic if there exists an isomorphism $\phi$ of $\Gamma$ into $\Delta$ such that

and

$$
\varphi(\gamma)=\psi(\phi \gamma)
$$

$$
\phi U=V \phi .
$$

Definition 1. Let $(\Gamma, \varphi)$ be an algebraic model for a von Neumann algebra $\mathcal{A}$ with a generating vector $x$ and $U$ an isomorphism of $\Gamma$ into $\Gamma$. Let $\alpha$ be an automorphism of $\mathcal{A}$. An algebraic ergodic system $(\Gamma, U, \varphi)$ is an algebraic model for $\alpha$ if

$$
J(U \gamma)=J(\gamma)^{\alpha} \quad \text { for } \quad \gamma \in \Gamma \text {, }
$$


where $J$ is an isomorphism of $\Gamma$ into $\Gamma(\mathcal{A})$ satisfying

$$
(J \Gamma)^{\prime \prime}=\AA
$$

and

$$
\varphi(\gamma)=(J \gamma x \mid x) \text { for } \gamma \in \Gamma .
$$

The following theorem is a nonabelian form of a theorem of Dinculeanu-Foiaş [3; Theorem 1]:

Theorem 1. Let $\mathcal{A}$ and $\mathscr{B}$ be von Neumann algebras acting cyclically on $\mathfrak{S}$ and $\Re$ respectively, $\alpha$ and $\beta$ be automorphisms of $\mathcal{A}$ and $\mathscr{B}$ respectively. If the algebraic models $(\Gamma, U, \varphi)$ for $\alpha$ and $(\Delta, V, \psi)$ for $\beta$ are isomorphic, then there exists an isomorphism $\Phi$ of $\mathcal{A}$ onto $\mathcal{B}$ such that $\Phi \circ \alpha=\beta \circ \Phi$.

Proof. Let $\Phi$ be a spatial isomorphism such that

$$
\Phi[J \gamma]=J(\phi \gamma) \quad \text { for } \quad \gamma \in \Gamma
$$

as in the proof of [6; Theorem 2], where $\phi$ is an isomorphism of $\Gamma$ onto $\Delta$ such that $\phi U=V \phi$. Since $J U=J^{\alpha}$ and $J V=J^{\beta}$, where $J^{\alpha}(\gamma)=J(\gamma)^{\alpha}$, then

$$
\begin{aligned}
\Phi \circ \alpha[J \gamma] & =\Phi\left[J^{\alpha} \gamma\right]=\Phi[J U \gamma]=J[\phi U \gamma]=J[V \phi \gamma] \\
& =J^{\beta}(\phi \gamma)=\beta \circ \Phi[J \gamma] \quad(\gamma \in \Gamma) .
\end{aligned}
$$

Since $(J \Gamma)^{\prime \prime}=\mathcal{A}$ and since $\alpha, \beta$ and $\Phi$ are ultraweakly continuous, we have $\Phi \circ \alpha=\beta \circ \Phi$.

3. When $(\Gamma, \varphi)$ is an algebraic model for $\mathcal{A}$, it is interesting to know what measure system related to $(\Gamma, \varphi)$ is an algebraic model for the crossed product $\mathcal{A} \otimes G$ of $\mathcal{A}$ by an automorphism group $G$ of $\mathcal{A}$. From this point of view, in this section we make an effort for getting response to the above question.

At first we shall briefly review the notion of the crossed product of von Neumann algebras introduced by [7]. Let $\mathcal{A}$ be a von Neumann algebra acting on $\mathfrak{S}_{\mathcal{E}}$ with a generating vector $x, G$ a countable group of automorphisms of $\mathcal{A}$. Choosing an othonormal base $\left(\delta_{\alpha}\right)_{\alpha \in G}$ of $l^{2}(G)$, where

$$
\delta_{\alpha}(\gamma) \begin{cases}=1 & \text { for } \gamma=\alpha, \\ =0 & \text { otherwise, }\end{cases}
$$

we can express every vector $y \in \mathfrak{S} \otimes l^{2}(G)$ in the form

where $\xi_{\beta} \in \mathfrak{S}$ and

$$
y=\sum_{\beta \in G} \xi_{\beta} \otimes \delta_{\beta},
$$

If we put

$$
\sum_{\beta \in G}\left\|\xi_{\beta}\right\|^{2}<+\infty
$$

$$
U_{\alpha}\left[\sum_{\beta \in G} \xi_{\beta} \otimes \delta_{\beta}\right]=\sum_{\beta \in G} U(\alpha) \xi_{\beta} \otimes \delta_{\alpha \beta},
$$

where $U(\alpha)$ is a unitary operator on $\mathfrak{B}$ determined by an algebraic model $(\Gamma, \varphi)$ for $\mathcal{A}$ and

$$
U(\alpha)[J(\gamma) x]=J(\gamma)^{\alpha-1} x \quad(\gamma \in \Gamma),
$$


then $U_{\alpha}$ is a unitary operator on $\mathscr{S}_{\mathcal{C}} \otimes l^{2}(G)$ which satisfies

and

$$
\begin{aligned}
U_{\alpha} U_{\beta} & =U_{\alpha \beta}, \\
U_{\alpha}^{*} & =U_{\alpha-1}
\end{aligned}
$$

$$
U_{\alpha}^{*}(a \otimes 1) U_{\alpha}=a^{\alpha} \otimes 1,
$$

for $a \in \mathcal{A}$ and $\alpha, \beta \in G$. Furthermore, we have

and

$$
\left(A U_{\alpha}\right)\left(B U_{\beta}\right)=A B^{\alpha-1} U_{\alpha \beta}
$$

$$
\left(A U_{\alpha}\right)^{*}=A^{* \alpha} U_{\alpha}^{*}
$$

where $A=a \otimes 1$ and $B=b \otimes 1$ for $a, b \in \mathcal{A}$. The crossed product $\mathcal{A} \otimes G$ of $\mathcal{A}$ by $G$ is the weak closure of

$$
\left\{\sum_{\alpha \in G}\left(a_{\alpha} \otimes 1\right) U_{\alpha} \mid a_{\alpha} \in \mathcal{A}\right\}
$$

on $\mathfrak{S} \otimes l^{2}(G)$.

Now suppose that the model satisfies
(*)
$(J \Gamma)^{\alpha} \subset J \Gamma$
for any $\alpha \in G$.

If we put

then $\gamma^{\alpha}$ is well-defined.

$$
\gamma^{\alpha}=J^{-1} \circ J^{\alpha}(\gamma) \quad \text { for } \alpha \in G \text { and } \gamma \in \Gamma,
$$

To proceed further, we shall need some lemmas.

Lemma 2. Let $G \times \Gamma$ be the Cartesian product of $G$ and $\Gamma$. If we define

$$
(\alpha, \gamma) \otimes(\beta, \delta)=\left(\alpha \beta, \gamma \delta^{\alpha-1}\right)
$$

for $\alpha, \beta \in G$ and $\gamma, \delta \in \Gamma$, then $(G \times \Gamma, \otimes)$ is a group.

Since the conclusion of the lemma follows from straightforward computations, we shall omit the detail. Hereafter, the group will be denoted by $G \otimes \Gamma$.

We define a complex-valued function $\psi$ on $G \otimes \Gamma$ by the following equation :

$$
\psi[(\alpha, \gamma)] \begin{cases}=\varphi(\gamma) & \text { if } \alpha=e, \\ =0 & \text { otherwise. }\end{cases}
$$

Then we have the following lemma:

Lemma 3. $\psi$ is a positive definite function on $G \otimes \Gamma$.

Proof. For any finite set of complex numbers $c_{1}, \cdots, c_{n}$ and $\left(\alpha_{1}, \gamma_{1}\right), \ldots,\left(\alpha_{n}, \gamma_{n}\right) \in G \otimes \Gamma$, we have

$$
\begin{aligned}
\sum_{i, j=1}^{n} & c_{i} c_{j}^{*} \psi\left[\left(\alpha_{i}, \gamma_{i}\right) \otimes\left(\alpha_{j}, \gamma_{j}\right)^{-1}\right] \\
& =\sum_{i, j=1}^{n} c_{i} c_{j}^{*} \psi\left[\left(\alpha_{i} \alpha_{j}^{-1}, \gamma_{i}\left(\gamma_{j}^{-1}\right)^{\alpha j \alpha_{i}^{-1}}\right)\right] \\
& =\sum_{i, j)} c_{i} c_{j}^{*} \varphi\left(\gamma_{i} \gamma_{j}^{-1}\right) \geqq 0,
\end{aligned}
$$

where $(i, j) \in\left\{(i, j) \mid \alpha_{i} \alpha_{j}^{-1}=e\right\}$.

By Lemmas 2 and 3 , we have

Lemma 4. $(G \otimes \Gamma, \psi)$ is a measure system. 
Now we can prove the following

Theorem 5. Let $(\Gamma, \varphi)$ be an algebraic model for a von Neumann algebra $\mathcal{A}$ with a generating vector $x$. If $G$ is a countable group of automorphisms of $\mathcal{A}$ satisfying the condition $(*)$, then $(G \otimes \Gamma, \psi)$ is an

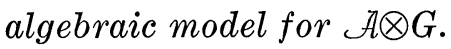

Proof. At first we define

$$
\tilde{J}[(\alpha, \gamma)]=(J(\gamma) \otimes 1) U_{\alpha}
$$

for $(\alpha, \gamma) \in G \otimes \Gamma$ (where $J$ is an isomorphism of $\Gamma$ into $\Gamma(\mathcal{A})$ determined by $(\Gamma, \varphi))$. Then we have

$$
\begin{aligned}
& \tilde{J}[(\alpha, \gamma) \otimes(\beta, \delta)]=\tilde{J}\left[\left(\alpha \beta, \gamma \delta^{\alpha-1}\right)\right] \\
&=\left(J\left(\gamma \delta^{\alpha-1}\right) \otimes 1\right) U_{\alpha \beta} \\
&=(J(\gamma) \otimes 1)\left(J(\delta)^{\alpha-1} \otimes 1\right) U_{\alpha} U_{\beta} \\
&=(J(\gamma) \otimes 1) U_{\alpha}(J(\delta) \otimes 1) U_{\beta} \\
&=\tilde{J}[(\alpha, \gamma)] \tilde{J}[(\beta, \delta)]
\end{aligned}
$$

for every $(\alpha, \gamma),(\beta, \delta) \in G \otimes \Gamma$, which shows that $J$ is a homomorphism

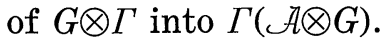

$\tilde{J}[(\alpha, \gamma)]=\tilde{J}[(\beta, \delta)]$ implies $J\left(\delta^{-1} \gamma\right) \otimes 1=U_{\beta \alpha-1}$ and choosing any orthonormal base $\left(e_{i}\right)_{i}$ of $\mathfrak{K}$, we have

$$
\left(J\left(\delta^{-1} \gamma\right) e_{i} \mid e_{j}\right)=\left(U\left(\beta \alpha^{-1}\right) e_{i} \mid e_{j}\right)\left(\delta_{\beta \alpha-1} \mid \delta_{e}\right)
$$

for any $i$ and $j$. If $\beta \alpha^{-1} \neq e$ then $\left(J\left(\delta^{-1} \gamma\right) e_{i} \mid e_{j}\right)=0$ for any $i$ and $j$, which is a contradiction. Therefore $\beta=\alpha$, or

$$
J\left(\delta^{-1} \gamma\right) \otimes 1=1
$$

which implies $\delta=\gamma$. Hence $\tilde{J}$ is one-to-one.

Since $(\tilde{J} \Gamma)^{\prime \prime}=\mathcal{A}$, we have $\tilde{J}[G \otimes \Gamma]=\mathcal{A} \otimes G$.

Furthermore, since $x \otimes \delta_{e}$ is a generating vector for $\mathcal{A} \otimes G$, we have

$$
\begin{aligned}
& \left(\tilde{J}[(\alpha, \gamma)] x \otimes \delta_{e} \mid x \otimes \delta_{e}\right)=\left((J(\gamma) \otimes 1)\left(U(\alpha) x \otimes \delta_{\alpha}\right) \mid x \otimes \delta_{e}\right) \\
& \quad=(J(\gamma) U(\alpha) x \mid x)\left(\delta_{\alpha} \mid \delta_{e}\right)= \begin{cases}(J(\gamma) x \mid x) & (\alpha=e) \\
0 & (\alpha \neq e)\end{cases} \\
& \quad=\psi[(\alpha, \gamma)]
\end{aligned}
$$

for any $(\alpha, \gamma) \in G \otimes \Gamma$. This completes the proof.

4. Let $(\Gamma, U, \varphi)$ be an algebraic model for an automorphism $\theta$ of an von Neumann algebra $\mathcal{A}$ with a generating vector $x$. Let $G, G \otimes \Gamma, \psi$ be as in Theorem 5 .

Now suppose that $\theta$ commutes with all elements of $G$. If we put

$$
\tilde{U}[(\alpha, \gamma)]=(\alpha, U \gamma) \text {, }
$$

then $U$ is an isomorphism of $G \otimes \Gamma$ into $G \otimes \Gamma$. Indeed,

$$
\tilde{U}[(\alpha, \gamma) \otimes(\beta, \delta)]=\left(\alpha \beta, U \gamma U\left(\delta^{\alpha-1}\right)\right) .
$$

Since $\theta$ commute with $G$, we have

$$
J\left[U\left(\delta^{\alpha-1}\right)\right]=J\left(\delta^{\alpha-1 \theta}\right)=J\left(\delta^{\theta-1}\right)=\left(J^{\theta}(\delta)\right)^{\alpha-1}=J\left[U(\delta)^{\alpha-1}\right] .
$$

Hence

Therefore

$$
U\left(\delta^{\alpha-1}\right)=(U \delta)^{\alpha-1} \quad \text { for any } \quad \alpha \in G .
$$




$$
\begin{aligned}
\tilde{U}[(\alpha, \gamma) \otimes(\beta, \delta)] & =\left(\alpha \beta, U \gamma(U \delta)^{\alpha-1}\right) \\
& =\tilde{U}[(\alpha, \gamma)] \otimes \tilde{U}[(\beta, \delta)],
\end{aligned}
$$

or $U$ is a homomorphism of $G \otimes \Gamma$ into $G \otimes \Gamma$. By the definition, $U$ is one-to-one.

Summing up, we have

Lemma 6. $(G \otimes \Gamma, \tilde{U}, \psi)$ is an algebraic ergodic system.

Conveniently, we shall identify $1 \otimes G$ with $\left\{U_{\alpha} \mid \alpha \in G\right\}$, cf. [1]. Under these circumstances, we shall show

Theorem 7. Let $(\Gamma, U, \varphi)$ be an algebraic model for an automorphism $\theta$ of a von Neumann algebra $\mathcal{A} ; G, G \otimes \Gamma, \psi$ as in Theorem 5 and

$$
\tilde{U}[(\alpha, \gamma)]=(\alpha, U \gamma) \quad((\alpha, \gamma) \in G \otimes \Gamma) .
$$

If $\theta$ commutes with all elements of $G$, then $(G \otimes \Gamma, \tilde{U}, \psi)$ is an algebraic model for $\theta \otimes 1$ which is an automorphism of $\mathcal{A} \otimes G$.

In the theorem, $\theta \otimes 1$ is defined as in [5] by

$$
\left.[J(\gamma) \otimes 1) U_{\alpha}\right]^{\theta \otimes 1}=\left(J^{\theta}(\gamma) \otimes 1\right) U_{\alpha}
$$

for every $\alpha \in G$ and $\gamma \in \Gamma$.

Proof. Let $\tilde{J}$ be an isomorphism of $G \otimes \Gamma$ into $\Gamma(\mathcal{A} \otimes G)$ as in Theorem 3. Then we have any $(\alpha, \gamma) \in G \otimes \Gamma$

$$
\begin{aligned}
\tilde{J} \tilde{U}[(\alpha, \gamma)] & =\tilde{J}(\alpha, U \gamma)=(J(U \gamma) \otimes 1) U_{\alpha} \\
& \left.=\left(J^{\theta}(\gamma) \otimes 1\right) U_{\alpha}=(J(\gamma) \otimes 1) U_{\alpha}\right)^{\otimes \otimes 1} \\
& =\tilde{J}^{\theta \otimes 1}[(\alpha, \gamma)] .
\end{aligned}
$$

Since the other part of the proof is clear, this completes the proof.

\section{References}

[1] H. Choda: On automorphisms of abelian von Neumann algebras. Proc. Japan Acad., 41, 280-283 (1965).

[2] N. Dinculeanu and C. Foiaş: Algebraic models for measures. Illinois J. Math., 12, 340-351 (1968).

[3] — : Algebraic models for measure preserving transformations. Trans. Amer. Math. Soc., 134, 215-237 (1969).

[4] J. Dixmier: Les algèbres d'opérateurs dans l'espace Hilbertien. GauthierVillars, Paris (1957).

[5] T. Saito: The direct and crossed product of rings of operators. Tohoku Math. J., 11, 299-304 (1959).

[6] H. Takai: On an algebraic model for von Neumann algebras. Proc. Japan Acad., 46, 941-943 (1970).

[7] T. Turumaru: Crossed product of operator algebras. Tohoku Math. J., 10, 355-365 (1958). 